\title{
Effects of Leached Amylose and Amylopectin in Rice Cooking Liquidon Texture and Structure of Cooked Rice
}

\author{
Liu Yang ${ }^{1,2}$; Yong-Hai Sun ${ }^{1}{ }^{*}$; Yang Liu ${ }^{1,3}$; Qian Mao ${ }^{1}$; Li-Xin You ${ }^{2}$; Ju-Min Hou ${ }^{1}$; \\ Muhammad Aqeel Ashraf ${ }^{4}$. \\ ${ }^{I}$ College of Food Science and Engineering,Jilin University, Changchun, China; ${ }^{2}$ College of Biology and Food, \\ Changchun Science and Technology University Changchun, China; ${ }^{3}$ School of Chemical and Biological \\ Technology, Jilin Institute of Chemical Technology, Jilin, China; ${ }^{4}$ Faculty of Science and Natural Resources, \\ University Malaysia, Sabah, Malaysia.
}

\begin{abstract}
Effects of leached amylose (AM) and amylopectin (AP) on textural and morphological properties of cooked rice were investigated separately by replacing cooking liquid with AM and AP separated from pouring cooking liquid. The pouring of cooking water reduced the hardness (from 28.45 to $19.42 \mathrm{~N}$ ) and stickiness (from 1.74 to $1.19 \mathrm{~N} \cdot \mathrm{s}$ ) significantly. However, the addition of $A M$ and AP enhanced the hardness (27.63N) and stickiness $(1.71 N \cdot s)$.Scanning electron microscopy show that the leached short-chain AM entered the surface hollows in the cooked rice after water evaporation. Meanwhile, the Long-chain AM cross-linked to formed a three-dimensional network structures, which covered on the filled hollows. This distribution led to a harder texture of cooked rice. The leached AP absorbed water and swelledto form masses. Atthe gelatinization temperature, theAPmassesagglomeratedtoformafilmlayer,whichcoveredtheunevenstructure, the thicker and smoother film contributed to the sticky texture.
\end{abstract}

Keywords: amylose, amylopectin, leached, morphological properties, textural properties

*Authors for correspondence: paperiset@163.com 


\section{INTRODUCTION}

Rice is recognized as a major cereal crop consumed in many countries, as it provides staple food for over half of the world's population, particularly in Asia. The textural properties of cooked rice are very important to its eating quality as they affect its acceptance by consumers (Wadaet al., 2006; Lyonet al., 2000). Texture is defined as a multidimensional property, while the most commonly-used parameters for cooked rice are hardness and stickiness (JulianoandPerez, 1983). Several factors related to textural quality have been identified, including AM content (Jinorose et al., 2014), unleachable AM content (Cameron and Wang, 2005), protein content (Salehand Meullenet, 2007; Mestres et al., 2011), fat content (Bileaderis and Tonogai, 1991), and the lengths of chains A and B in AP(Patindol et al., 2010). Several research groups have associated the leached materials (LMs) with the textural changes and eating quality. When cooked at the optimum water level, rice grains absorb water and swell greatly, leading to the leach of some materials into the cooking water, and more than $90 \%$ of the leached solids are M and AP (Hanashiro et al., 2004). The leached AM and short-chain AP during cooking are the main indicators of hardness and stickiness of cooked rice (Ongand Blanshard, 1995a). The ratio of the leached AM-AP contributes to the hardness and stickiness of cooked rice (Patindolet al., 2010). The proportion of AP in the leached starch significantly and positively influences overall eating quality, and it is reduced as the proportion of AM in leached starch increases (Wada et al., 2010; Hanashiroet al., 2004). The leached AM and AP are gelatinized and coated onto the surface of cooked grains as a coated layer by boiling (Tamura and Ogawa, 2012), contributing to the improvement of rice eating quality (WADA et al. 2010). The highquality cooked grains exhibit a thicker and smoother surface (Okuda et al., 2009).

To our knowledge, many studies focus on the effects of leached AM and AP on textural properties of the cooked rice, but little information has been provided about the reasons. Therefore, the objectives of this study are to observe the distribution of the leached AM and AP on the surface of the cooked rice (forming process of coated layer) by scanning electron microscopy
(SEM), and thereby to reveal the effects of the leached AM and AP on hardness and stickiness.

\section{MATERIALS AND METHODS}

MaterialsMilled Rice grains of Dao Huaxiang were used in the experiments, this variety was grown in Wuchang, Heilongiang province, China, was purchased from a local rice store, and stored at $4^{\circ} \mathrm{C}$ in a refrigerator before experiment.

Leaching of the total solids (TSs)About $10 \mathrm{~g}$ of rice was soaked with distilled water (rice/water ratio $=1: 2$ ) in a beaker at $20{ }^{\circ} \mathrm{C}$ for $30 \mathrm{~min}$ and then cooked on a heating pad, the cover of the beaker was equipped with a thermometer. The cooking liquid was heated from 20 to $100^{\circ} \mathrm{C}$. Nine samples were cooked at a time and the experiments were conducted twice. The cooking liquid was poured and filtered when the temperature reached $20,30,40,50,60,70,80,85$ or $90^{\circ} \mathrm{C}$. The first cooking water was reserved, while the second was separated into water-soluble contents (supernatant fraction) and water-insoluble contents (precipitate fraction) by centrifugation at $4000 \mathrm{rpm}$ for $10 \mathrm{~min}$. The amount of TSs in separated cooking water were measured by ovendrying at $105^{\circ} \mathrm{C}$ for $12 \mathrm{~h}$.

Leaching of $A M$ and $A P$ The experiments were conducted twice again following the above cooking method. The separated cooking water, precipitate and supernatant at each temperature were freeze-dried for $12 \mathrm{~h}$. The freeze-dried powder was shattered, defatted with absolute ether and deprived of ethylether. The amounts of leached AM and AP were measured using a dual wavelength iodine binding technique (Fan et $a l ., 2008)$. The leached AM and AP were expressed as content of AM and AP leached per $1 \mathrm{~mL}$ cooking water.

Chemical composition of the rice cooking water $\left(85^{\circ} \mathrm{C}\right)$ The experiments was conducted again following the above cooking method. The poured cooking water at $85^{\circ} \mathrm{C}$ was freeze-dried and the composition were analyzed.The crude protein, lipid, and ash contents were determined using the standard AACC (2000) methods. Apparent AM (AAM) and AP were determined by the dual wavelength colorimetric method. The total starch and true AM content ( TAM) were determined following approved method AACC (2000) using a total starch assay kit and an amylopectin/amylose assay kit (Megazyme; Wicklow; Ireland), 
respectively. The amount of the leached TSs was measured by oven-drying at $105^{\circ} \mathrm{C}$ for $12 \mathrm{~h}$. All calculated based on the amount of the leached solids.And all measurements were done in triplicate.

Separation and purification of the leached AM and $A P$ The cooking liquid frozen powder was defatted, then protein was removed. The leached TSs may be further fractionated with n-butanol into AM and the AP using crystallization with nbutanol and isoamylol repeatedly (Duet al., 1998). Then quite pure AM and AP were obtained after freeze-drying.

Gel filtration chromatography50 $\mathrm{mg}$ separated starch, AM and AP were dissolved in $1 \mathrm{~mL}$ absolute ethyl alcohol and $2 \mathrm{~mL}$ water, $3 \mathrm{~mL}$ $2 \mathrm{~mol} / \mathrm{L} \mathrm{NaOH}$ was added and vibrated fully. $5 \mathrm{~mL}$ water was added gradually and continued to vibrate until the sample were dissolved completely, the $\mathrm{pH}$ was adjusted to 6.5-7 with $\mathrm{HCl}$, deionizedwater was added to make exactly $25 \mathrm{~mL}$, the concentration of the solution was $2 \mathrm{~mol} / \mathrm{L}$, then filtered through a filter membrane (pore size $0.24 \mu \mathrm{m}$ ), from which $4 \mathrm{~mL}$ was injected into Sepharose CL-2B chromatographiccolumn $(1.7 \times 75 \mathrm{~cm})$ and eluted with $50 \mathrm{mmol} / \mathrm{L} \mathrm{NaCl}$ at a flow rate of $16 \mathrm{~mL} / \mathrm{h}$. The eluant was collectted with automatic sampling instrument, $4 \mathrm{~mL}$ in each tube, from which $1 \mathrm{~mL}$ was measured at $490 \mathrm{~nm}$ with phenol-sulfuric acid method (Shinluand Cheng, 1996).

Characteristic of the isolated AM and APThe content of $\mathrm{AM}$ and $\mathrm{AP}$ in the isolated $\mathrm{AM}$ and $\mathrm{AP}$ were measured using the dual wavelength colorimetric method, Crude protein, lipids and ash contents were determined according to AACC methods. Blue value and $\lambda_{\max }$ were determined according to the method described by Suzukiet al. (1981). The iodine affinities (IA, g/100 g) of AM and AP were determined by the automatic potentiometric titrator method (Schoch, 1964).

Light microscopyLight microscopy study was carried out according toLiuet al.,(2013). About 0.1 $\mathrm{mg}$ of the purified AM, AP, freeze-dried cooking liquid powder were transferred into a $2 \mathrm{~mL}$ tube with a stopple separately, the $0.2 \%$ iodine in $2 \%$ potassium iodide diluted $2: 1$ with distilled water $\left(\mathrm{KI} / \mathrm{I}_{2}\right)$ was used to stain starch, and then observed and photographed in the bright field using an Olympus BX51 light microscope (Olympus Corporation, Japan).

Replacing rice cooking liquidThe experimental method according to Hanashiro et al., ( 2004 ) with some modification. $10 \mathrm{~g}$ of rice was cooked at room temperature according to the above cooking methods, and after arriving at $85^{\circ} \mathrm{C}$, the cooking liquid was poured out entirely until no liquid flew out. Then the mass of the cooking liquid, the water content, the dry matter contents of both AM and AP were computed. Each experiment was repeated five times and the average was used as the final result. Under the same conditions, another 8 samples were cooked. For each sample, after the temperature reached $85^{\circ} \mathrm{C}$, the cooking liquid was poured out entirely. An equal amount of the $85^{\circ} \mathrm{C}$ water in the cooking liquid was used to dissolve the $1 / 3 \times, 2 / 3 \times$, or $1.0 \times a m o u n t$ of leached AM, $1 / 3 \times, 2 / 3 \times$, or $1.0 \times a m o u n t$ of leached AP, or 1.0xamount of leached AM and AP, separately. These solutions were poured into the above 7 samples separately as the replacement of cooking liquid, while the 8 th sample was filled with an equal amount of $85^{\circ} \mathrm{C}$ water from the cooking liquid. The mixtures were blended uniformly under gentle stirring with a glass stick. Then the samples were cooked for 20 min and stewed for $40 \mathrm{~min}$. Each sample was tested in quintuplicate. The cooking liquid of the last reference sample was not replaced.

Texture analysisTextural properties of cooked rice were analyzed with a texture analyzer (Stable Micro System, TA.XT.Plus, Surrey, UK) by the uniaxial compression method. Three intact cooked rice kernels were placed on a flat aluminum plate (100 $\mathrm{mm}$ diameter) and a cylindrical probe with a diameter of $50 \mathrm{~mm}$ was used to compress the kernels to $85 \%$ deformation. Pre-test speed, test speed and post-test speed of plunger were set at 1.0, 1.0 and $10 \mathrm{~mm} / \mathrm{s}$. Measuredparameters according to Rewthong, (2011). A force-time curve was obtained from the test and the following textural parameters were determined.

hardness - the maximum compressive force during extrusion $(\mathrm{N})$,

stickiness - area under the curve (N.s).

Scanning electron microscopy (SEM)The morphological characteristics of the cooked rice under various treatment conditions was examined by SEM (XL30 ESEM-FEG, FEL COMPANY, Finland) at $10 \mathrm{kV}$. The cooked rice was freezedried to have a final moisture lower than $5 \%$. Samples were attached to a SEM stub using a double-backed cellophane tape and then coated with gold-palladium, the outer surface of the samples was observed and photographed. 
Statistical analysis Analysis of variance was performed with the SPSS program version 13.0 and was presented as mean values with standard deviations. Differences between mean values were established using Duncan's multiple range tests at a confidence level of $95 \%$. All experiments were performed in triplicate.

\section{RESULTS AND DISCUSSION}

Leaching characteristic ofTSs, AM and APFigure 1 shows the change of leached TSs in the cooking liquid under varying temperature.The temperature and the amount of TSs were in linear relation, and more TSs leached with the temperature rise. The hydration of the rice kernels was increased with temperature rise, which facilitated the leaching of carbohydrates (Hanand Lim, 2009). At lower than $40^{\circ} \mathrm{C}$, the TSs in the cooking liquid were mainly the water-insoluble portion, whereas the amount of the water-soluble andthe water-insolubleportion increased significantly since $50^{\circ} \mathrm{C}$ and $70^{\circ} \mathrm{C}$, respectively. The leachate were maximized at $85^{\circ} \mathrm{C}$ and then decreased with the temperature rise. At $85^{\circ} \mathrm{C}$. The leached materials were almost water-insoluble, constituting the main solids in the cooking liquid.

Figures $2 \mathrm{a}$ and $2 \mathrm{~b}$ show the leaching properties of $\mathrm{AM}$ and $\mathrm{AP}$ of rice grains under different cooking temperatures. The data showed that $96.19 \%$ of the leached TSs were AM and AP. As reported, although only AM was leached into the cooking water during cooking, while AP was left in the gelatinized granules (Juliano and Perez, 1983), several previous studies confirmed that not only AM but AP leached during cooking (Cameron and Wang, 2005; Patindol et al., 2007). The data showed that the leached $\mathrm{AM}$ (before $40^{\circ} \mathrm{C}$ ) was almost water-insoluble at relatively low temperature. As it reached $50^{\circ} \mathrm{C}$, the $\mathrm{AM}$ began to dissolve and the amount of water-soluble AM increased significantly. The leached AP was almost water-insoluble,which increased significantly from $70^{\circ} \mathrm{C}$.From this point, a higher temperature led to the leach-out of more AP. The amount of leached AM and AP was maximized at $85^{\circ} \mathrm{C}$, which is the gelatinized temperature of starch (Wadaet al., 2010), and then decreased with the temperature rise. The main proportion of the leached starch was AP (64.29\%), which was more than two times of AM (31.84\%).

The structural of starch in rice grains was showed in Figure.3a. Starch granules are heterogeneous mixtures of two kinds of molecule, AM and AP. $\mathrm{AM}$ is a smaller component and an essentially lineara-1,4-glucan which contains only tiny amounts of a-1,6-branch linkages, while AP is a larger component and a brancheda-1,4-anda-1,6glucan. AM chains adopt helical structures and AP presented cluster-like structures (Fig.3b and Fig.3e) (Mottiar and Altosaar, 2011). In the center of a starch grain, the long-chain AM can bind with the external links of AP to form double-helix structures, the granule periphery is highly organized by short-chain of AM and AP clusters, which constitutes the framework of starch grains (Fig.3a) (Naguleswaran et al., 2014; Oates, 1997). The starch molecules in rice are tightly distributed to form fascircular micella, which are unpenetrable to water molecules. During soaking and cooking, the AM and AP located near the surface of the rice could leach out into water at low temperature (Han and Lim, 2009). Along with temperature rise, water permeated into the partially crystallized AM and AP molecules in rice grains, also the starch grains started to absorb water and swell, the starch grains broke down to form unimolecule, and the doublehelix of AM was opened, then AM and AP in starch grains were leached gradually (Janeand Shen,1993). The AM was usually leached earlier than the AP (Hizukuri, 1991).

When water was finally absorbed or evaporated, the leached materials formed a coated layer by condensing and coating onto the surface of cooked grain (Tamura and Ogawa, 2012), leading to the loss of the AM and AP in the cooking liquid.

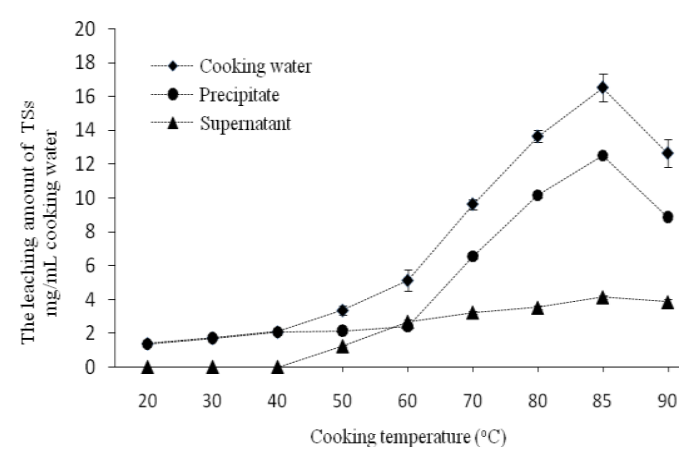


Fig.1. The amount of leached TSs in rice cooking liquid during cooking under different temperatures
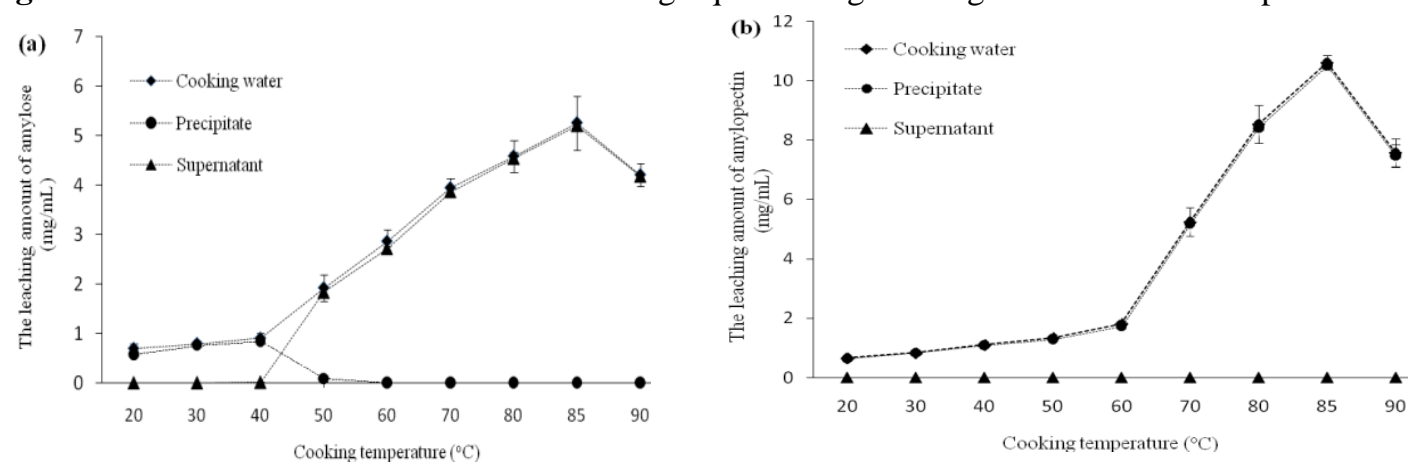

Fig.2.The amount of leached amylose and amylopectin in rice cooking liquid during cooking under different temperatures.

(a) amylose, (b) amylopectin

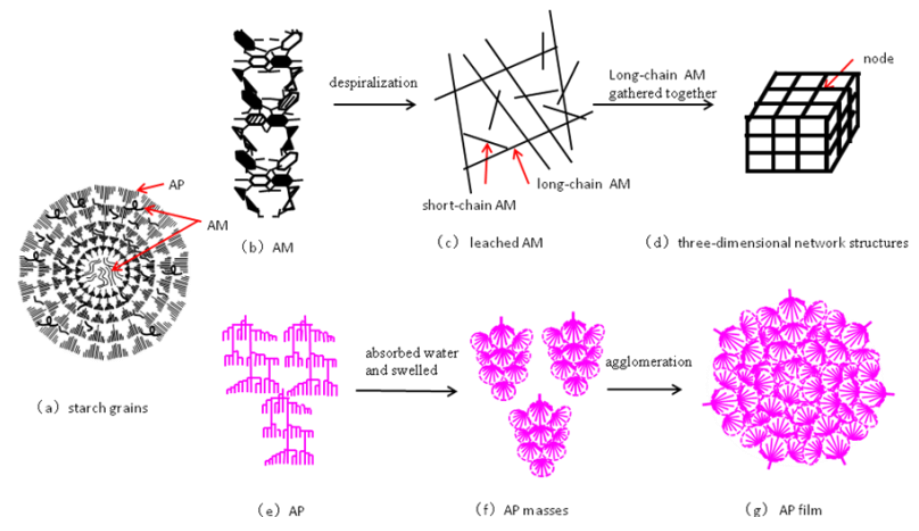

Fig.3. Schematic representation of starch grain structure andchanging of leached AM and AP during cooking

Composition of the rice cooking water(at $85^{\circ}$ )Table 1 summarizes the chemical composition of the rice cooking water at $85^{\circ} \mathrm{C}$. The leached solids essentially consisted of starch (95.54\%), a minimal amount of protein, and traces of lipid, ash $(2.1 \%, 1.23 \%, 0.84 \%)$. The result accorded with the report of Patindol et al.,(2007).The content of AAM and TAM were $32.17 \%$ and $31.55 \%$ respectively, whereas the leached AP was $63.27 \%$. These firmed previous findings that both AM and AP leached out of rice grains during cooking(Cameron and Wang, 2005; Patindolet al.,2007). The tiny differences in the content of TAM and AAM also indicated that the intermediate material in starch was left in grains. The result accorded with the reports of Patindolet al., (2010); Patindol and Wang, (2002), who confirmed that the leached starch consists of two components during rice cooking: $\mathrm{AM}$ and $\mathrm{AP}$ by HPSEC.

Table 1.Chemical composition of the rice cooking water at $85^{\circ} \mathrm{C}(\%$, dry base).

\begin{tabular}{cc}
\hline Sample & cooking water $\left(85^{\circ} \mathrm{C}\right)$ \\
\hline TSs $(\mathrm{g} / 100 \mathrm{~g}$ of rice $)$ & 1.4 \\
Total starch $(\%)$ & 94.54 \\
AAM $(\%)$ & 32.17 \\
TAM $(\%)$ & 31.55 \\
AP $(\%)$ & 63.27 \\
Crude protein $(\%)$ & 2.1 \\
Crude lipid $(\%)$ & 1.23 \\
Crude ash $(\%)$ & 0.84 \\
Others $(\%)$ & 1.29 \\
\hline
\end{tabular}


Data are mean of 3 replicates.

Characteristic of the isolated $A M$ and APGel chromatography shows that the AM and AP after separation and purification both show a main peak and a few of weak peaks (Figure 4). These impure peaks may be some unseparated AM, AP or impurities. The ratio of impure-peakarea is quite small compared to total area, which confirmed that the isolated AM and AP were quite pure.

The chemical composition of the isolated AM and AP are presented in table 2. The purity of the AM and AP were judged on the basis of composition

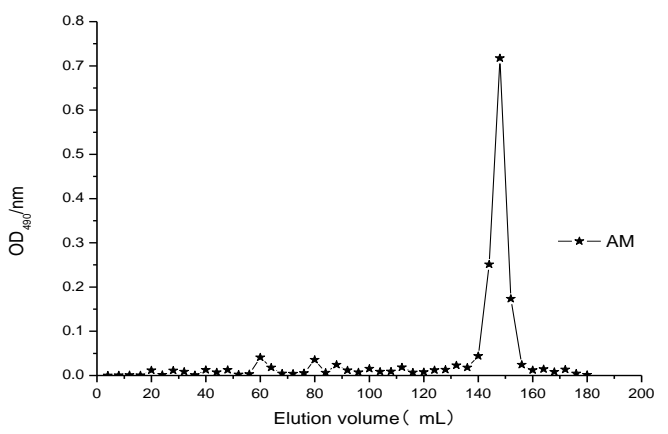

high AM (92.23\%) and high AP (93.41\%) and low lipid $(0.28 \%, 0.32 \%)$, low protein $(0.4 \%, 0.35 \%)$ and trace amount of ash $(0.2 \%, 0.42 \%)$. The blue values of isolated AM and AP were 0.12 and 1.14 respectively, whereas the maximumabsorptionwavelength $(\lambda \max )$ of the AM and $\mathrm{AP}$ are $608 \mathrm{~nm}$ and $536 \mathrm{~nm}$ respectively. Iodine affinities were 18.45 and 0.52 respectively. These parameter values indicated a high purity of isolated AM and AP.

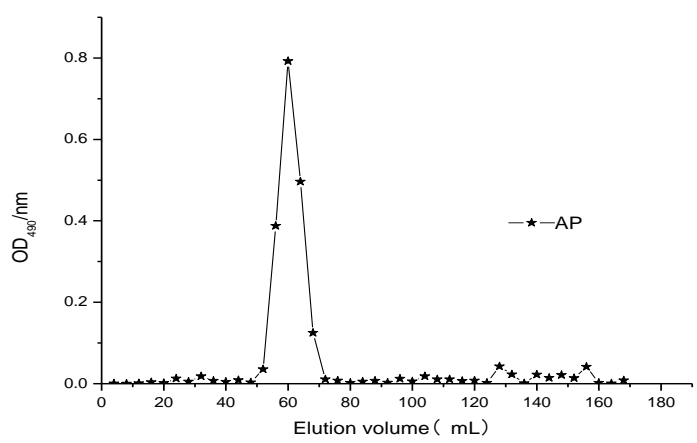

Fig.4.Gel filtration elution curves of separated AM (a) and AP (b).

\begin{tabular}{lcc}
\hline \multicolumn{1}{c}{ Sample } & Isolated AM & Isolated AP \\
\hline Chemical component & 92.23 & \\
AM $(\%)$ & 5.37 & 4.2 \\
AP $(\%)$ & 0.4 & 93.41 \\
Crude protein $(\%)$ & 0.28 & 0.35 \\
Crude lipid $(\%)$ & 0.20 & 0.32 \\
Crude ash $(\%)$ & 1.52 & 0.42 \\
Others $(\%)$ & & 1.3 \\
Properties & 18.45 & \\
Iodine affinity $(\mathrm{g} / 100 \mathrm{~g})$ & 1.12 & 0.52 \\
Blue value & 608 & 0.14 \\
$\lambda$ max $(\mathrm{nm})$ & & 536 \\
\hline
\end{tabular}

Table 2. Chemical composition and characteristics of the isolated AM and AP (\%, dry base)

Data are mean of 3 replicates.

Optical microscopy of the isolated AM and AP Optical microscopy revealed obvious differences in $\mathrm{KI} / \mathrm{I}_{2}$ staining and morphology between the leached AM and AP. The AM stained uniformly blue with $\mathrm{KI} / \mathrm{I}_{2}$ in all fields and formed a threedimensional network structures by cross-linking, uniformly scattering in the rice cooking liquid (Fig.5a). Whereas the AP created a purple-red colour by staining and showed unregular masses structure (Fig.5b). The unsegregated starch stained hyacinthine uniformly and the swelled AP imbedded in a continuous matrix of cross-linked AM molecules (Fig.5c) (Lii et al., 1995). 
Effects of Amylose and Amylopectin of Rice

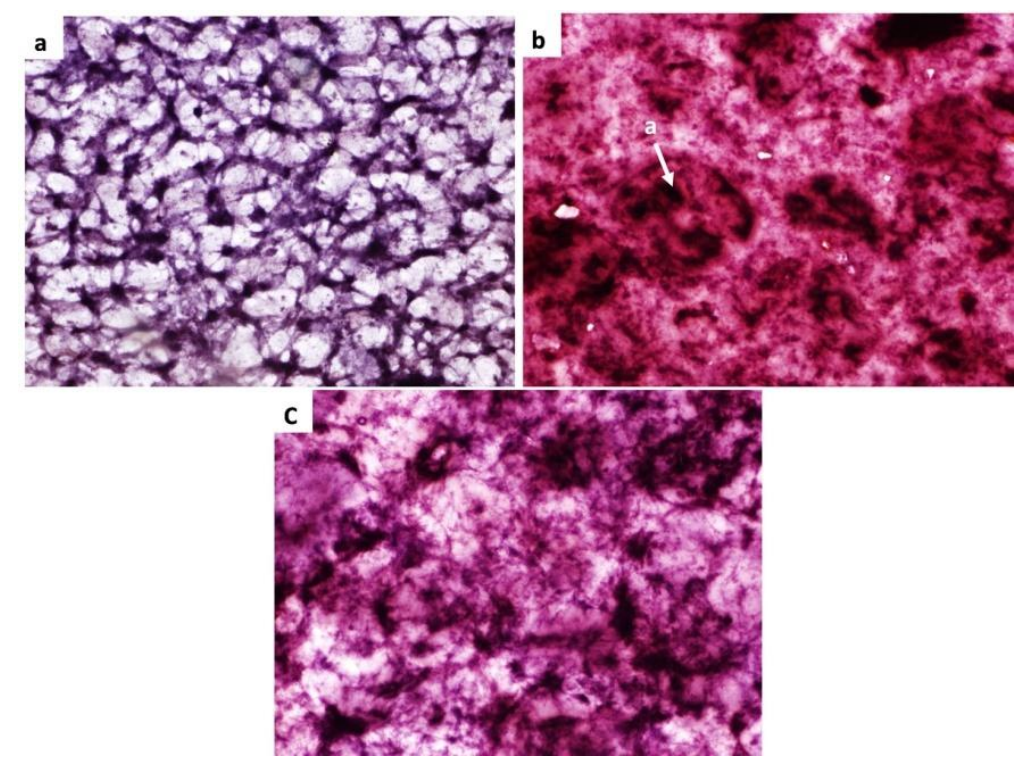

Fig 5. KI/I $\mathrm{I}_{2}$ stained purified (a)AM,(b) AP,(c) starch in the cooking liquid (a: APmass).

Textural properties The results of textural properties of the cooked rice including hardness and stickiness are shown in Table 3. Compared to the normally-cooked grains (NR), the replacement of the cooking liquid at $85^{\circ} \mathrm{C}$ with distilled water (RW) resulted in the significant reduction of both hardness (from 28.45 to $19.42 \mathrm{~N}$ ) and stickiness (from 1.74 to $1.19 \mathrm{~N} \cdot \mathrm{s}$ ). The coated layer was confirmed to be correlated with the textural qualities of cooked rice (Wada et al., 2010). Therefore, replacement of the cooking liquid with distilled water led to the loss of the leached carbohydrate, which then impacted the formation of coated layers, resulting in lower hardness and stickiness.

The leached AM as a replacement obviously change the hardness value from that of sample RW (hardness: $19.42 \pm 1.35$ vs. 27.63 \pm 3.31 ), but were similar to those of samples NR (hardness: $28.45 \pm 2.89$ vs. $27.63 \pm 3.31)$. The amount of leached AM positively influenced the hardness of cooked rice. Although the hardness of cooked rice is more closely correlated with unleachable AM than with the leached AM (Cameron and Wang, 2005), a larger amount of leached AM is related to higher hardness of cooked rice (Han and Lim, 2009). However, the sample 1RTIN show similar stickiness with samples NR (Stickiness: 1.71 \pm 0.27 vs. $1.74 \pm 0.1$ ), but were higher than those of samples RW (Stickiness: $1.71 \pm 0.27$ vs.1.19 \pm 0.12 ). The leach-out of more AP led to higher stickiness, indicating that the leached AP might contribute to the stickiness of the cooked rice. This illustrated that the amount and components of leached starch are associated with different textural characteristics of cooked rice (Testerand Morrison, 1990).

When the cooking water was replaced with $1 \mathrm{RSE}$ and 1RTIN, the texture properties were rather identical with sample NR.

Table 3.Summary of textural changes of cooked rice samples after different replacements of cooking water

\begin{tabular}{llc}
\hline Substitutes of cooking water & Hardness ( N ) & Stickiness( N.s ) \\
\hline NR & $28.45 \pm 2.89^{\mathrm{a}}$ & $1.74 \pm 0.1^{\mathrm{a}}$ \\
RW & $19.42 \pm 1.35^{\mathrm{b}}$ & $1.19 \pm 0.12^{\mathrm{b}}$ \\
1/3RSE & $22.35 \pm 2.34^{\mathrm{bc}}$ & $1.18 \pm 0.15^{\mathrm{b}}$ \\
2/3RSE & $24.81 \pm 2.26^{\mathrm{c}}$ & $1.20 \pm 0.18^{\mathrm{b}}$ \\
1RSE & $27.63 \pm 3.31^{\mathrm{a}}$ & $1.21 \pm 0.15^{\mathrm{b}}$ \\
1/3RTIN & $20.26 \pm 2.0^{\mathrm{b}}$ & $1.47 \pm 0.35^{\mathrm{ac}}$ \\
2/3 RTIN & $19.5 \pm 1.92^{\mathrm{b}}$ & $1.57 \pm 0.31^{\mathrm{a}}$ \\
1 RTIN & $20.43 \pm 2.32^{\mathrm{b}}$ & $1.71 \pm 0.27^{\mathrm{a}}$ \\
1 RSE and 1RTIN & $27.65 \pm 2.65^{\mathrm{a}}$ & $1.70 \pm 0.14^{\mathrm{a}}$ \\
\hline
\end{tabular}

Mean values \pm standards $(n \geqq 5)$. Different letters within the same column indicate significant difference $(\mathrm{p}<0.05) . N R$ : The cooking liquid was not replaced; RW: replacement with distilled water; 
1/3RSE, 2/3RSE、1RSE: replacement with 1/3x、2/3 $\times 、 1 \times$ amount of leached AM; 1/3RTIN, 2/3 RTIN,1RTIN: replacement with $1 / 3 \times, 2 / 3 \times, 1 \times$ amount of leached AP; 1RSE and 1RTIN: replacement with $1.0 \times a m o u n t$ of leached AM and AP.

Microstructure.Figure.6 shows the surface microstructure of the cooked rice after different treatments. Under the normal rice cooking condition, the SEM displays an uneven structure covered by the formed films (Fig.6a). Sample RW exhibits a number of irregular large hollows (Fig.6b). The appearance of the hollows can be explained by the fact that during cooking, the starch located at the surface could leach out easily into water, resulting in high void density at the exterior surface (Rewthonget al., 2011). Additionally, the internally stored starch absorbs water through voids and swells greatly, disrupting the cell walls beneath the surface (Horigane et al.,1999; Ogawa et al., 2003). These hollows facilitate the leach-out of AM and AP located in the inner areas, leading to the formation of denser and larger hollows near the exterior surface of the cooked rice. The morphological properties of cooked rice correspond to the texture properties. After the AM and AP diffuses out of the swollen granules and leached into the cooking water, the starch granules disrupted and lose rigidity. The framework of rice grains lose its support, and the structure of rice grains become very loose, which results in the formation of a fluffy, soft and nonsticky texture of cooked rice (Lu et al., 2013).

The microstructures of samples RSE are illustrated in Fig.6d-f. The addition of AM seemingly led to the filling of surface hollows in the cooked rice (Fig.6d), the hollows were filled up gradually with the increased addition of AM (Fig.6e). After filling up the hollows, the appearance of cooked rice displays an uneven structure (Figure 6f).

The leached AM in cooking water is relatively smaller and greater mobility than AP, which facilitated themselves entering into the hollows at the surface of the cooked rice (Han and Lim, 2009). When the water was finally boiled off, the leached short-chain AM began to migrate and entered into the hollows at the surface of the cooked rice. In the meantime, the long-chain AM cross-linked and formed a three-dimensional network structures via hydrogen bond gradually (Fig.3d) (Naguleswaranet al.,2014; Zhaoet al., 2001; Mitchell and Ledward, 1986). The structures covered on the filled hollows. After the hollows on surface of rice were filled up,the remaining shortchain AM is bound to the nodes on the reticular
structures(Fig.3d) (Zhao et al., 2001), which leads to the formation of uneven starch body structures on the surfaces of the cooked rice (Fig.6f).

The main framework of rice is starch grains, while AP also forms the framework of starch grains (Fitzgerald, 2004). The entered AM binds via hydrogen bond with the unleachable hydroxy on the side chains of AP, which functions as the filling framework (Fitzgerald, 2004). Maybe all these chains participate in intermolecular interaction, thus affecting the rigidity of the starch granule and hence indirectly led the harder cooked rice texture (Laura et al., 2006). Whereas the thicker AM body covered on the filled hollows further strengthened the intermolecular forces and retrograded rapidly, contributing to the harder and compacted physical structure of cooked rice ( $\mathrm{Yu}$ et al.,2009; Patindol et al., 2010).

The SEM images of samples RTIN exhibit a smooth, solid and porous film structure, (Fig.6i).The cluster-like AP in starch granule absorbed water and swelled, leading to volume swelling and structural loosening(Fig.3f), followed by AP leaching when starch granules disrupted eventually. The leached AP formed a viscous solution (Yehand Li, 1996). Because of viscosity, the AP was not fully dispersed in water, which led to the formation of segmental masses by crosslinking (Fig.5b). The masses were scattered in the rice cooking liquid unregularly. After water evaporation, agglomeration occurred between AP masses owing to the surface phenomenon and the adsorption by grains, a thick layer of sticky AP film was formed and covered the large hollows on the surface of rice(Fig.3g) (Zhaoet al., 2001). However, the amount of the leached AP was insufficient to form thicker films to cover the hollows completely, resulting in the formation of a net-like film structure. Figure $6 \mathrm{~g}$ exhibits the thinnest film and the largest pore size. With the increased addition of AP, the films gradually became thicker and smoother, while the pore size was gradually diminished (Fig.6h). Stickiness was defined as the cohesion strength at a surface level is thought to be an important parameter of cooked rice quality. The stickiness of cooked rice was correlated with the amount of coated layer (Ikeda, 2001), since a thicker and smoother film led to higher stickiness, which accorded with the textural 
properties. As reported, a higher quality of cooked grains corresponds to the thicker and smoother surface (Okuda et al., 2009).

When the cooking water was replaced by 1 xamount of AM and AP, the sample showed the similar microstructure with samples NR. A thicker and smoother film covered uneven starch body as the coated layer of the cooked rice (Fig.6c), which should have included AM filled into the voids, covered on the surface in addition to the film created by AP (Tamuraand Ogawa, 2012), contributing to the texture properties of the cooked rice.
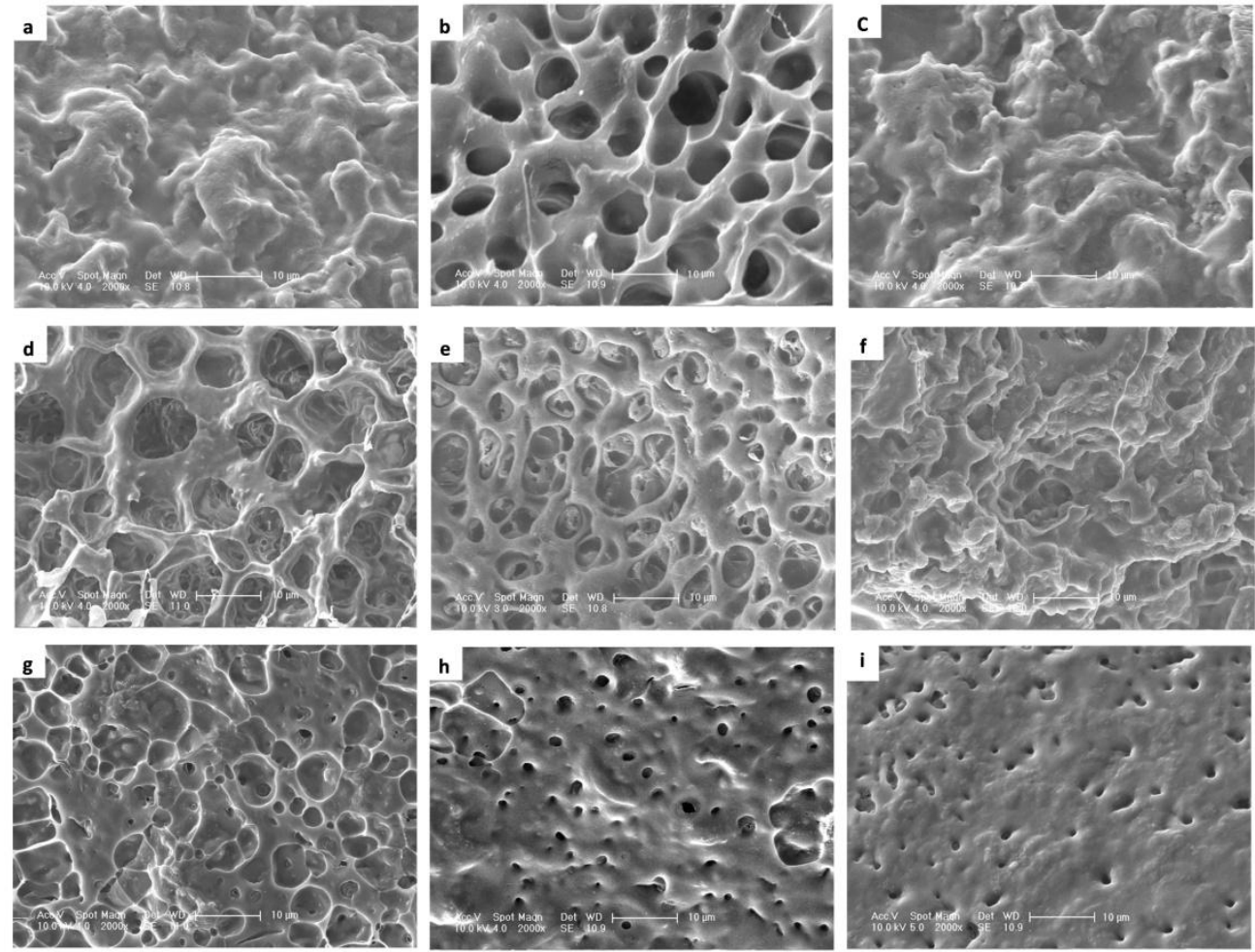

Fig.6.Scanning electron micrograms of the outer surface of cooked rice.(a) NR, (b) RW, (c) 1RSE and 1RTIN, (d) 1/3RSE, (e) 2/3RSE, (f) 1RSE, (g) 1/3RTIN, (h) 2/3RTIN, (i) 1RTIN

\section{CONCLUSIONS}

This study revealed the effects of the leached AM and $\mathrm{AP}$ on the textural and morphological properties of cooked rice. The leached AM improved the hardness of cooked rice, whereas the leached AP contributed to the sticky texture, which corresponded to the observed morphology. As the water reduction, the leached short-chain AM entered into the hollows at the surface of the cooked rice. Meanwhile, the Long-chain AM cross-linked to formed a three-dimensional network structures. The network structures covered on the filled hollows.After the hollows were filled up,the remaining short-chain AM is bound to the nodes on the reticular structures, which leads to the formation of uneven starch body structures. The entered AM formed union zones stabilized by hydrogen bonds with the unleachable chains of AP and strengthened the rigidity of the starch granule, thus indirectly led to the harder cooked rice texture. Whereas the covered AM body at the surface of the cooked rice retrograded rapidly and further increased the hardness of cooked rice. Because of viscosity, the leached AP formed segmental masses by crosslinking. The masses agglomerated to form a film layer after water evaporation, covering the uneven structure. The thicker and smoother film contributed to the sticky texture.

\section{ACKNOWLEDGMENTS}

This study was supported by National Natural Science Foundation of China (NNSFC, No. 31271861); Jilin Provincial Science \& Technology Department (No. 201205013); Jilin Provincial Research Foundation ofEducation Departmentthe 12th Five-Year 
Plan( No.【2011】239号) and Changchun University of Science and Technology.

\section{REFERENCES}

1. Wada, M., Tsubone, Y., Hamanhi and Ogata, T. (2006). Evaluation and use of physicochemical properties as index trains for selecting rice cultivars with extremely high palatability. Jpn. J. Crop Sci, 75, 38-43.

2. Lyon, B.G., Champagne, E.T., Vinvard, B.T., and Windham, W.R. (2000). Sensory and instrumental relationships of texture of cooked rice from selected cultivars and postharvest handling practices. Cereal Chem, 77, 64-69.

3. Lu, S., Cik, T.T., Lii, C.Y., Lai, P., and Chen, H.H. (2013). Effect of amylose content on structure, texture and $\alpha$-amylase reactivity of cooked rice. $L W T$ Food Science and Technology,54, 224-228.

4. Jane, J., and Shen, J.J. (1993). Internal structure of the Potato starch granule revealed by Chemical gelatinization. Carbohydres Res, 247, 279290.

5. Juliano, B.O., Perez C.M. (1983). Major factors affecting cooked milled rice hardness and cooking time. J. Texture Stud,14, 235-243.

6. Jinorose, M., Prachayawarakorn, S., and Soponronnari, S. (2014). A novel image-analysis based approach to evaluate some physicochemical and cooking properties of rice kernels. J .Food Eng,124, 184-190.

7. Naguleswaran, S., Vasanthan, T., Hoover, R., and Bressler, D. (2014). Amylolysis of amylopectin and amylose isolated from wheat, triticale. Food Hydrocolloids, 35, 686-693.

8. Cameron, D.K., and Wang, Y.J. (2005). A better understanding of factors that affect the hardness and stickiness of long-grain rice. Cereal Chem, 82, 113119.

9. Saleh, M.I., and Meullenet, J.F. (2007). Effect of protein disruption using proteolytic treatment on cooked rice texture properties. J. Texture Stud,38, 423437.

10. Mestres, C., Ribbeyre, F., Pons, B., Fallet, V., and Matencio, F. (2011). Sensory texture of cooked rice is rather linked to chemical than to physical characteristics of raw grain. J Cereal Sci, 53, 81-89.

11. Mottiar, Y., and ALTOSAAR, I. (2011). Iodine sequestration by amylose to combat iodine deficiency disorders.Trends Food Sci Tech, 22, 335340.

12. Mitchell, J.R., and Ledward, D.A. (1986). Functional Properties of Food Macromolecules. Elsevier applied science publishers LTD, London, 121198.

13. Biliaderis, G.C., and Tonogai, J.R. (1991). Influence of lipids on the thermal and mechanical properties of concentrated starch gels. J. Agric. Food Chem,39, 833-840.

14. Patindol, J., Gu X., and Wang, Y.J. (2010). Chemometric analysis of cooked rice texture in relation to starch fine structure and leaching characteristics. Starch-Starke, 62, 188-197.

15. Patindol, J.A., Gonzalez, B.C., Wang, Y.J., and Mcclung, A.M. (2007). Starch fine structure and physicochemical properties of specialty rice for canning. J Cereal Sci, 45, 209-218.

16. Patindol, J., Wang, Y.J. (2002). Fine structures of starches from long-grain rice cultivars with different functionality. Cereal Chem, 79, 465-469.

17. Lii, C.Y., Shao, Y.Y., and Tseng, K.H. (1995). Gelation mechanism and rheological properties of rice starch. Cereal Chem, 71, 393-400.

18. Hanashiro, I., Ohta, K., Takeda, C., and Mizukami, H. (2004). Leaching of amylose and amylopectin during Cooking of Rice Grains and Their effect on adhesiveness of cooked Rice. J. Appl. Glycoscience, 51, 349-354.

19. Ong, M.H., and Blanshard. (1995a). Texture determinants of cooked, parboiled rice. I. Rice starch amylose and the fine structure of amylopectin. J. Cereal Sci,21, 251-260.

20. Oates, C.G. (1997). Towards an understanding of starch granule structure and hydrolysis. Trends Food Sci Tech, 8, 375-382.

21. Wada, T., Umemoto, T., Aoki, N., Tsubone, M., Ogata, T., and Kondo, M. (2010). Starch Eluted from Polished Rice during Soaking in Hot Water is Related to the Eating Quality of Cooked Rice. Journal of Applied Glycoscience, 58, 13-18.

22. Tamura, M., and Ogawa, Y. (2012). Visualization of the coated layer at the surface of rice grain cooked with varying amounts of cooking water. $J$. Cereal Sci,,56, 404-409.

23. Tester, R.F., and Morrison, W.R. (1990). Swelling and gelatinization of cereal starches. Cereal Chem,67, 558-563.

24. Okuda, R., Ishimura, T., and Kanatani, A. (2009). Study on rice cooking, part 1: effect of solid components in the rice cooking liquid on the rice grain surface. Journal of Cookery Science of Japan, 42, 394403.

25. Ogawa, Y., Glenn, G.A., Orts, W.J., and Wood, D.F. (2003). Histological structures of cooked rice grain. J Agr Food Chem, 51, 7019-7023.

26. Fan, M., Zhang, C., Zhang, Q., and Li, X. (2008). Determination of amylose and amylopectin content in sorghum by dual wavelength method. China Brew, 21, 85-87.

27. Du, X.F., XU, S.Y., and Wang, Z. (1998). Isolationandpurification of amylase and amylopectin in pueraria lobata. Food and Fermentation Industries, 4, $18-21$.

28. Shinlu, C.Y.C., and Cheng,Y.(1996). GelChromatography Fractionation and Thermal 
Characterization of Rice Starch Affected by Hydrothermal Treatment. Cereal Chem.73, 5-11.

29. Suzuki, A., Hizukuri, S., and Takeda, Y. (1981). Physicochemical studies of kuzu starch. Cereal Chem, 58, 286-290.

30. Schoch, T. J. (1964). Iodimetric determination of amylose. Potentiometric titration: Standard method. London: Academic Press, 157-160.

31. Liu, D., Parker, M.L., Wellner, N., Kirby, A.R., Cross, K., Morris, V.J., and Cheng, F. (2013). Structural variability between starch granules in wild type and in ae high-amylose mutant maize kernels. Carbohydr Polym,97, 458-68.

32. Rewthong, O., Soponronnarit, S., Taechapairoj, C., Tungtrakul, P., and Prachayawarakorn, S. (2011). Effects of cooking, drying and pretreatment methods on texture and starch digestibility of instant rice. J.Food Eng,103, 258-264.

33. Han, J.A., and Lim, S.T. (2009). Effect of Presoaking on Textural, Thermal, and Digestive Properties of Cooked Brown Rice. Cereal Chem,86, 100-105.

34. Hizukuri, S. (1991). Properties of hot-waterextractable amylose. Carbohydrate Res, 217, 251-253.

35. Horigane, A.K., Toyoshima, H., Hemmi, H., Engelaar, W., OKUBO, A., and Nagata, T. (1999). Internal hollows in cooked rice grains observed by NMR micro imaging. J Food Sci, 64, 1-5.

36. Zhao, S.M., Xiong, S.B., and Zhang, S.H. (2001). Paste System on Rice Starch and Its Retrogradation Properties. Journal of the Chinese Cereals and Oils Association, 16, 19-21.

37. Fitzgerald, M., (2004). Starch in Rice chemistry and technology, E.T. ChamPagne, Editor. American Association of Cereal Chemists: St. Paul, MN, U.S.A.

38. Laura B. I., Beatriz L.M., and Maria C.A. (2006). Effect of amylose on starch pastes viscoelasticity and cooked grains stickiness in rice from seven argentine genotypes. Food Res Int, 39, 660-666.

39. Yu, S.F., Ma, Y., and Sun, D.W. (2009), Impact of amylose content on starch retrogradation and texture of cooked milled rice during storage. J. Cereal Sci, 50, 139-144.

40. Yeh, A.I., Li J.Y. (1996). A Continuous Measurement of Swelling of Rice Starch During Heating. J. Cereal Sci, 23, 277-283.

41. Ikeda, H., (2001). Relationship between the saccharides extracted from rice grains during cooking and the sensory taste of cooked rice. Journal of Home Economics of Japan, 52, 401-409. 